\title{
THE QUADRATIC FORM IN THE LÉVY-KHINCHIN FORMULA ON SEMIGROUPS
}

\author{
DRAGU ATANASIU
}

(Communicated by Palle E. T. Jorgensen)

\begin{abstract}
In this paper we obtain the quadratic form in the Lévy-Khinchin formula on a commutative involutive semigroup, with a neutral element, as a sum of two simpler quadratic forms.
\end{abstract}

The Lévy-Khinchin representation for a negative definite function, with the real part bounded below, defined on a commutative involutive semigroup with a neutral element, was proved in [1, p. 108, Theorem 3.19] .

In Section 2 of this paper we give a proof for this Lévy-Khinchin representation in which we obtain the quadratic form $q$ from [1] as a sum of an additive function and a quadratic form associated with a bi-additive function.

Section 3 is concerned with negative definite functions which have real part bounded below, defined on the semigroup $\left(\mathbb{Z}^{2},+\right)$ with the involution $(m, n)^{*}=$ $(n, m)$. We obtain the Lévy-Khinchin representation of these functions using the result of Section 2.

\section{Notation}

Let $(S,+, *)$ be a commutative involutive semigroup with neutral element ([1, p. 86]). We say that a function $\varphi: S \rightarrow \mathbb{C}$ is positive definite if for each natural number $n \geq 1$, each family $c_{1}, \ldots, c_{n}$ of complex numbers and each family $x_{1}, \ldots, x_{n}$ of elements of $S$, we have

$$
\sum_{j, k=1}^{n} c_{j} \bar{c}_{k} \varphi\left(x_{j}+x_{k}^{*}\right) \geq 0
$$

A function $\varphi: S \rightarrow \mathbb{C}$ is hermitian if $\varphi\left(x^{*}\right)=\overline{\varphi(x)}$ for each $x \in S$.

We say that an hermitian function $\varphi: S \rightarrow \mathbb{C}$ is negative definite if for each natural number $n \geq 2$, each family $c_{1}, \ldots, c_{n}$ of complex numbers such that $c_{1}+$ $\ldots+c_{n}=0$, and each family $x_{1}, \ldots, x_{n}$ of elements of $S$ we have

$$
\sum_{j, k=1}^{n} c_{j} \bar{c}_{k} \varphi\left(x_{j}+x_{k}^{*}\right) \leq 0
$$

Received by the editors November 7, 1996.

1991 Mathematics Subject Classification. Primary 43A35; Secondary 60B15.

Key words and phrases. Negative definite function, involutive semigroup, Radon measure, Lévy-Khinchin formula, quadratic form.

(C) 1998 American Mathematical Society 
We denote by $\Gamma$ the set

$$
\left\{\rho: S \rightarrow \mathbb{C}\left|\rho(x+y)=\rho(x) \rho(y) ; \rho\left(x^{*}\right)=\overline{\rho(x)} ;\right| \rho(x) \mid \leq 1 ; \rho(0)=1\right\}
$$

and by $\Omega$ the set $\{\rho \in \Gamma \mid \rho \not \equiv 1\}$.

With the product topology $\Gamma$ is a compact space and $\Omega$ a locally compact space.

We denote by $\mathcal{M}(S)$ the set of positive Radon measure on $\Omega$ such that the functions $(\rho \mapsto 1-\operatorname{Re} \rho(x))_{x \in S}$ are $\mu$-integrable for each $\mu$ in $\mathcal{M}(S)$.

An element of $\mathcal{M}(S)$ is called a Lévy measure. We denote by $\mathcal{Q}(S)$ the set

$$
\left\{q: S \rightarrow\left[0, \infty\left[\mid 2(q(x)+q(y))=q(x+y)+q\left(x+y^{*}\right), x, y \in S\right\} .\right.\right.
$$

An element of $\mathcal{Q}(S)$ is called a quadratic form on $S$. This notion of quadratic form was introduced in [5, p. 211].

Let $\mathcal{A}(S)$ be the set

$$
\left\{a: S \rightarrow\left[0, \infty\left[\mid a(x+y)=a(x)+a(y), a\left(x^{*}\right)=a(x), x, y \in S\right\}\right.\right.
$$

and $\mathcal{B}(S)$ the set

$$
\begin{aligned}
& \left\{b: S \times S \rightarrow \mathbb{R} \mid b(x, y)=b(y, x), b\left(x^{*}, y\right)=-b(x, y),\right. \\
& b(x+y, z)=b(x, z)+b(y, z), b(x, x) \in[0, \infty[, x, y, z \in S\} .
\end{aligned}
$$

We denote by $\mathcal{L}(S)$ the set

$$
\begin{aligned}
\{L: S & \times \Omega \rightarrow \mathbb{R} \mid L(x+y, \rho)=L(x, \rho)+L(y, \rho), L\left(x^{*}, \rho\right)=-L(x, \rho), x, y \in S ; \\
& \rho \mapsto L(x, \rho) \text { is continuous on } \Omega, x \in S ; \\
& \rho \mapsto L(x, \rho)-\operatorname{Im} \rho(x) \text { is } \mu \text {-integrable, } x \in S, \mu \in \mathcal{M}(S)\} .
\end{aligned}
$$

An element of $\mathcal{L}(S)$ is called a Lévy function. In [4] is proved that the set $\mathcal{L}(S)$ is nonvoid.

Let $\mathcal{T}(S)$ be the set

$$
\left\{\ell: S \rightarrow \mathbb{R} \mid \ell(x+y)=\ell(x)+\ell(y), \ell\left(x^{*}\right)=-\ell(x), x, y \in S\right\} .
$$

\section{The LÉVy-KhinChin REPRESEntation}

Theorem. For a function $\varphi: S \rightarrow \mathbb{C}$ the following conditions are equivalent:

(i) the function $\varphi$ is negative definite and has real part bounded below;

(ii) for every $L \in \mathcal{L}(S)$ there are $C \in \mathbb{R}, a \in \mathcal{A}(S), b \in \mathcal{B}(S), \ell \in \mathcal{T}(S)$ and $\mu \in \mathcal{M}(S)$ which satisfy

$$
\varphi(x)=C+a(x)+b(x, x)+i \ell(x)+\int_{\Omega}(1-\rho(x)+i L(x, \rho)) d \mu(\rho), x \in S .
$$

$C, a, b$ and $\mu$ are uniquely determined by $\varphi ; \ell$ is uniquely determined by $\varphi$ and $L$. We have the relations

$$
a(x)=\lim _{n \rightarrow \infty} \frac{\varphi\left(n\left(x+x^{*}\right)\right)}{2 n} \quad \text { and } \quad b(x, x)=\lim _{n \rightarrow \infty} \frac{\operatorname{Re} \varphi(n x)}{n^{2}}, x \in S .
$$

Proof. Let $U$ be the vector space

$$
\left\{f: \Gamma \rightarrow \mathbb{R} \mid f(\rho)=\sum_{k=1}^{n} a_{k} \rho\left(x_{k}\right), \sum_{k=1}^{n} a_{k}=0, n \in \mathbb{N}, n \geq 2, a_{k} \in \mathbb{C}, x_{k} \in S\right\}
$$

and $U_{+}=\{f \in U \mid f \geq 0\}$.

For every $t \in] 0, \infty\left[\right.$ the function $\psi_{t}: S \rightarrow \mathbb{C}$ defined by $\psi_{t}(x)=e^{-t \varphi(x)}$ is positive definite (cf. [1, p. 74, Theorem 2.2]) and bounded. 
It follows from [1, p. 93, Theorem 2.5] that for each $t \in] 0, \infty[$ there is a positive Radon measure $\mu_{t}$ on $\Gamma$ such that

$$
e^{-t \varphi(x)}=\int_{\Gamma} \rho(x) d \mu_{t}(\rho)
$$

Let $n \geq 2$ be a natural number, $a_{1}, \ldots, a_{n}$ complex numbers such that $a_{1}+\ldots+a_{n}=$ 0 , and $x_{1}, \ldots, x_{n}$ elements of $S$.

We have

$$
\sum_{k=1}^{n} a_{k}\left(\frac{e^{-t \varphi\left(x_{k}\right)}-1}{t}\right)=\frac{1}{t} \int_{\Gamma} \sum_{k=1}^{n} a_{k} \rho\left(x_{k}\right) d \mu_{t}(\rho) .
$$

Letting $t$ tend to 0 , we obtain that the function $F: U \rightarrow \mathbb{R}$ defined by

$$
F\left(\rho \mapsto \sum_{k=1}^{n} a_{k} \rho\left(x_{k}\right)\right)=-\sum_{k=1}^{n} a_{k} \varphi\left(x_{k}\right)
$$

is well defined. If in (1) we take $x_{k}+y$ instead of $x_{k}$ and assume that the function $\rho \mapsto \sum_{k=1}^{n} a_{k} \rho\left(x_{k}\right)$ is in $U_{+}$, we obtain, also letting $t$ tend to 0 , that the function

$$
y \mapsto-\sum_{k=1}^{n} a_{n} \varphi\left(x_{n}+y\right)
$$

is positive definite and bounded.

Consequently Theorem 2.5 from [1] , p. 93, implies that for every $g \in U_{+}$there is a positive Radon measure $\mu_{g}$ on $\Gamma$ such that if $g(\rho)=\sum_{k=1}^{n} a_{k} \rho\left(x_{k}\right), \sum_{k=1}^{n} a_{k}=0$, we have

$$
-\sum_{k=1}^{n} a_{k} \varphi\left(x_{k}+y\right)=\int_{\Gamma} \rho(y) d \mu_{g}(\rho), y \in S .
$$

Let $f \in U, g \in U_{+}$. We suppose that

$$
f(\rho)=\sum_{k=1}^{n} a_{k} \rho\left(x_{k}\right), \sum_{k=1}^{n} a_{k}=0 \quad \text { and } \quad g(\rho)=\sum_{\ell=1}^{m} b_{\ell} \rho\left(y_{\ell}\right), \sum_{\ell=1}^{m} b_{\ell}=0 .
$$

We have

$$
F(f g)=-\sum_{k=1}^{n} \sum_{\ell=1}^{m} a_{k} b_{\ell} \varphi\left(x_{k}+y_{\ell}\right)=\int_{\Gamma} f(\rho) d \mu_{g}(\rho) .
$$

This implies that if $h, g \in U_{+}$we have

$$
F(\rho \mapsto \rho(x) h(\rho) g(\rho))=\int_{\Gamma} \rho(x) h(\rho) d \mu_{g}(\rho)=\int_{\Gamma} \rho(x) g(\rho) d \mu_{h}(\rho), x \in S,
$$

and consequently that

$$
h \mu_{g}=g \mu_{h} .
$$

It follows from (3) that we can define a positive Radon measure $\mu$ on $\Omega$ such that

$$
\left.\mu\right|_{O_{g}}=\left.\frac{1}{\left.g\right|_{O_{g}}} \mu_{g}\right|_{O_{g}}
$$

where $O_{g}=\{\rho \in \Gamma \mid g(\rho)>0\}$.

It is easy to verify that

$$
\left.\mu_{g}\right|_{\Omega}=\left.g\right|_{\Omega} \mu
$$


The measure $\mu$ is the Lévy measure for $\varphi$ (cf. [1, p. 103, 3.12]).

Using (4), for $g$ in $U_{+}$we obtain

$$
\int_{\Omega} g(\rho) d \mu(\rho)=\int_{\Omega} d \mu_{g} \leq \int_{\Gamma} d \mu_{g}=F(g) .
$$

We denote by $E$ the set

$$
\left\{f \in U \mid F(f)=\int_{\Omega} f(\rho) d \mu(\rho)\right\} .
$$

The relations (2) and (4) yield

$$
f g \in E \quad \text { for } \quad f \in U \quad \text { and } \quad g \in U_{+}
$$

because $f(\theta)=0$, where $\theta: S \rightarrow \mathbb{R}$ is identically 1 .

We denote by $B: S \times S \rightarrow \mathbb{R}$ the function defined by

$$
B(x, y)=F(\rho \mapsto \operatorname{Im} \rho(x) \operatorname{Im} \rho(y))-\int_{\Omega} \operatorname{Im} \rho(x) \operatorname{Im} \rho(y) d \mu(\rho) .
$$

It is immediate that

$$
B\left(x^{*}, y\right)=-B(x, y) \quad \text { and } \quad B(x, x) \geq 0 .
$$

It follows from (6) that the functions

$$
\rho \mapsto(1-\operatorname{Re} \rho(x)) \operatorname{Im} \rho(y) \operatorname{Im} \rho(z)
$$

and

$$
\rho \mapsto(1-\operatorname{Re} \rho(y)) \operatorname{Im} \rho(x) \operatorname{Im} \rho(z)
$$

are in $E$.

The equality

$$
\begin{aligned}
& F(\rho \mapsto(1-\operatorname{Re} \rho(x)) \operatorname{Im} \rho(y) \operatorname{Im} \rho(z)+(1-\operatorname{Re} \rho(y)) \operatorname{Im} \rho(x) \operatorname{Im} \rho(z)) \\
& =\int_{\Omega}((1-\operatorname{Re} \rho(x)) \operatorname{Im} \rho(y) \operatorname{Im} \rho(z)+(1-\operatorname{Re} \rho(y)) \operatorname{Im} \rho(x) \operatorname{Im} \rho(z)) d \mu(\rho)
\end{aligned}
$$

is equivalent to

$$
B(x+y, z)=B(x, z)+B(y, z) \quad x, y, z \in S .
$$

We have proved that $B \in \mathcal{B}(S)$.

Define $a: S \rightarrow \mathbb{R}$ by

$$
\begin{aligned}
& a(x)=-\varphi(0)+\operatorname{Re} \varphi(x)-\frac{1}{2} B(x, x)-\int_{\Omega}(1-\operatorname{Re} \rho(x)) d \mu(\rho) \\
& =F\left(\rho \mapsto 1-\operatorname{Re} \rho(x)-\frac{1}{2}(\operatorname{Im} \rho(x))^{2}\right)-\int_{\Omega}\left(1-\operatorname{Re} \rho(x)-\frac{1}{2}(\operatorname{Im} \rho(x))^{2}\right) d \mu(\rho) .
\end{aligned}
$$

First we have $a\left(x^{*}\right)=a(x)$.

Using the relations

$$
\begin{aligned}
& 1-\operatorname{Re} \rho(x)-\frac{1}{2}(\operatorname{Im} \rho(x))^{2} \\
& =\frac{1}{2}(1-\operatorname{Re} \rho(x))^{2}+\frac{1}{2}\left(1-(\operatorname{Re} \rho(x))^{2}-(\operatorname{Im} \rho(x))^{2}\right) \geq 0,
\end{aligned}
$$

we see from (5) that $a(x) \geq 0$ for every $x \in S$.

The fact that the function

$$
\rho \mapsto(1-\operatorname{Re} \rho(x))(1-\operatorname{Re} \rho(y))
$$


is in $E$ yields

$$
\begin{aligned}
B(x, y) & =F(\rho \mapsto \operatorname{Im} \rho(x) \operatorname{Im} \rho(y)-(1-\operatorname{Re} \rho(x))(1-\operatorname{Re} \rho(y))) \\
& -\int_{\Omega}(\operatorname{Im} \rho(x) \operatorname{Im} \rho(y)-(1-\operatorname{Re} \rho(x))(1-\operatorname{Re} \rho(y))) d \mu(\rho) \\
& =F(\operatorname{Re} \rho(x)+\operatorname{Re} \rho(y)-1-\operatorname{Re} \rho(x+y)) \\
& -\int_{\Omega}(\operatorname{Re} \rho(y)+\operatorname{Re} \rho(x)-1-\operatorname{Re} \rho(x+y)) d \mu(\rho) .
\end{aligned}
$$

Using (7) and the equality

$$
B(x, y)=\frac{1}{2}(B(x+y, x+y)-B(x, x)-B(y, y)),
$$

we obtain

$$
a(x+y)=a(x)+a(y), \quad x, y \in S .
$$

We have proved that $a \in \mathcal{A}(S)$.

The function $\ell: S \rightarrow \mathbb{R}$ defined by

$$
\ell(x)=\operatorname{Im} \varphi(x)-\int_{\Omega}((-\operatorname{Im} \rho(x))+L(x, \rho)) d \mu(\rho)
$$

satisfies

$$
\ell\left(x^{*}\right)=-\ell(x) .
$$

We have

$$
\begin{aligned}
& \operatorname{Im} \varphi(x)+\operatorname{Im} \varphi(y)-\operatorname{Im} \varphi(x+y) \\
& =\int_{\Omega}(\operatorname{Im} \rho(x+y)-\operatorname{Im} \rho(x)-\operatorname{Im} \rho(y)) d \mu(\rho)
\end{aligned}
$$

because the function

$$
\begin{aligned}
\rho & \mapsto \operatorname{Im} \rho(x)+\operatorname{Im} \rho(y)-\operatorname{Im} \rho(x+y) \\
& =(1-\operatorname{Re} \rho(x)) \operatorname{Im} \rho(y)+(1-\operatorname{Re} \rho(y)) \operatorname{Im} \rho(x)
\end{aligned}
$$

is in $E$. Consequently the function $\ell$ also satisfies

$$
\ell(x+y)=\ell(x)+\ell(y) .
$$

Taking $b(x, y)=\frac{1}{2} B(x, y)$, we finish the proof of the implication (i) $\Rightarrow$ (ii).

(ii) $\Rightarrow$ (i). Let $b \in \mathcal{B}(S)$. Using the relation

$$
b\left(x+y^{*}, x+y^{*}\right)=b(x, x)+b(y, y)-2 b(x, y)
$$

we see, as in [1, p. 103], that the function $x \mapsto b(x, x)$ is negative definite. Now the implication (ii) $\Rightarrow$ (i) is clear.

If we have a representation as in (ii), we obtain

$$
\begin{gathered}
\varphi\left(n\left(x+x^{*}\right)\right)=\varphi(0)+2 n a(x)+n^{2} b\left(x+x^{*}, x+x^{*}\right) \\
+\int_{\Omega}\left(1-\rho\left(n\left(x+x^{*}\right)\right) d \mu(\rho), \quad x \in S, n \in \mathbb{N}^{*} .\right.
\end{gathered}
$$

The dominated convergence theorem implies

$$
\lim _{n \rightarrow \infty} \frac{1}{2 n} \int_{\Omega}\left(1-|\rho(x)|^{2 n}\right) d \mu(\rho)=0,
$$

because $\frac{1-|\rho(x)|^{2 n}}{2 n} \leq 1-|\rho(x)|^{2}$. 
The relations $b\left(x+x^{*}, x+x^{*}\right)=0,(8)$ and (9) yield

$$
a(x)=\lim _{n \rightarrow \infty} \frac{1}{2 n} \varphi\left(n\left(x+x^{*}\right)\right) .
$$

From the equality (7) we obtain

$$
\begin{aligned}
& 2 b\left(n x, n x^{*}\right)=\varphi(0)-\varphi(n x)-\varphi\left(n x^{*}\right)+\varphi\left(n\left(x+x^{*}\right)\right) \\
& +\int_{\Omega} 1-\left((\rho(x))^{n}\right)\left(1-\left(\rho\left(x^{*}\right)\right)^{n}\right) d \mu(\rho)
\end{aligned}
$$

We have, using again the dominated convergence theorem,

$$
\lim _{n \rightarrow \infty} \frac{1}{n^{2}} \int_{\Omega}\left(1-(\rho(x))^{n}\right)\left(1-\left(\rho\left(x^{*}\right)\right)^{n}\right) d \mu(\rho)=0
$$

because $\frac{1}{n^{2}}\left|\left(1-(\rho(x))^{n}\right)\left(1-\left(\rho\left(x^{*}\right)\right)^{n}\right)\right| \leq|1-\rho(x)|^{2}$.

The relations $b\left(x, x^{*}\right)=-b(x, x),(10),(11)$ and (12) now give

$$
b(x, x)=\lim _{n \rightarrow \infty} \frac{1}{n^{2}} \operatorname{Re} \varphi(n x) .
$$

The representation from (ii) implies that

$$
\begin{aligned}
& \varphi(x+y)+\varphi\left(x+y^{*}\right)-2 \varphi(x) \\
& =2\left(a(y)+b(y, y)+\int_{\Omega} \rho(x)(1-\operatorname{Re} \rho(y)) d \mu(\rho)\right),
\end{aligned}
$$

which proves the unicity of the measure $\mu$. This completes the proof of the theorem.

Corollary. A function $q: S \rightarrow[0, \infty[$ is an element of $\mathcal{Q}(S)$ if and only if there are $a \in \mathcal{A}(S)$ and $b \in \mathcal{B}(S)$ such that

$$
q(x)=a(x)+b(x, x) .
$$

The functions $a$ and $b$ are uniquely determined by $q$ according to the relations

$$
a(x)=\frac{1}{2} q\left(x+x^{*}\right) \quad \text { and } \quad b(x, x)=\lim _{n \rightarrow \infty} \frac{q(n x)}{n^{2}}, x \in S .
$$

The corollary is an immediate consequence of the unicity results in the theorem of this section and [1, p. 108, Theorem 3.19]. It also results from [1] , p. 102, because if we denote by $a: S \rightarrow \mathbb{R}$ the function defined by

$$
a(x)=q(x)-b(x, x),
$$

where $b(x, y)=\frac{1}{2}(-q(x)-q(y)+q(x+y))$, we have

$$
a(x+y)=a(x)+a(y), a\left(x^{*}\right)=a(x),
$$

and the definition of $\mathcal{Q}(S)$ gives

$$
a(x)=q(x)-\frac{1}{2}(-2 q(x)+q(2 x))=\frac{1}{2} q\left(x+x^{*}\right) .
$$

Remark 1. Using the method of Bloom and Ressel from [2], we deduce from the representation given in this paper that the quadratic form on some commutative hypergroups may be written as a sum of two quadratic forms (see [2, p. 248, Theorem 2.4, and p. 250, Theorem 2.6]).

Remark 2. The relation $q(x)=a(x)+b(x, x)$ of the corollary is also a consequence of $[3$, p. 636, Theorem 8]. 


\section{An APPLicATion}

Proposition. Consider on the semigroup $\left(\mathbb{Z}^{2},+\right)$ the involution $(m, n)^{*}=(n, m)$. For a function $\varphi: \mathbb{Z}^{2} \rightarrow \mathbb{C}$ the following conditoins are equivalent:

(i) the function $\varphi$ is negative definite and has real part bounded below;

(ii) there are real numbers $C, \alpha, \beta, \gamma$, such that $\alpha, \beta \geq 0$, and a positive Radon measure $\mu$ on $\{z \in \mathbb{C}|| z \mid=1, z \neq 1\}$ such that the function $z \mapsto 1-\operatorname{Re} z$ is $\mu$-integrable, which satisfy

$$
\begin{aligned}
\varphi(m, n) & =C+(m+n) \alpha+(m-n)^{2} \beta+i(m-n) \gamma \\
& +\int_{T \backslash\{1\}}\left(1-z^{m} \bar{z}^{n}+(m-n) \operatorname{Im} z\right) d \mu(z),
\end{aligned}
$$

where $T=\{z \in \mathbb{C}|| z \mid=1\}$.

$C, \alpha, \beta, \gamma$ and $\mu$ are uniquely determined by $\varphi$.

Proof. We note that the function

$$
z \mapsto\left((m, n) \mapsto z^{m} \bar{z}^{n}\right)
$$

is a homeomorphism of $T$ onto the space of bounded characters of $\mathbb{Z}^{2}$.

It is easy to see that $a \in \mathcal{A}\left(\mathbb{Z}^{2}\right)$ if and only if there is $\alpha \in[0, \infty[$ such that $a(m, n)=(m+n) \alpha$, that $b \in \mathcal{B}\left(\mathbb{Z}^{2}\right)$ if and only if there is $\beta \in[0, \infty[$ such that $b((m, n),(p, q))=(m p+n q-m q-n p) \beta$, and that $\ell \in \mathcal{T}\left(\mathbb{Z}^{2}\right)$ if and only if there is a real number $\gamma$ such that $\ell(m, n)=(m-n) \gamma$.

Let $\mu$ be a positive Radon measure on $T \backslash\{1\}$ such that the function $z \mapsto 1-\operatorname{Re} z$ is $\mu$-integrable. We note that the function $z \mapsto(1-z)^{2}$ is also $\mu$-integrable.

We show that the functions $z \rightarrow 1-z^{m} \bar{z}^{n}+m(z-1)+n(\bar{z}-1)$ and $z \mapsto$ $1-\operatorname{Re} z^{m} \bar{z}^{n}$ are $\mu$-integrable for every $(m, n) \in \mathbb{Z}^{2}$. Take, for example, $m<0$ and $n \geq 0$. Using the binomial theorem, we obtain that the function

$$
\left(\frac{1}{z}\right)^{-m} \bar{z}^{n}+m\left(\frac{1}{z}-1\right)-n(\bar{z}-1)-1
$$

is $\mu$-integrable.

We have

$$
\begin{aligned}
& z^{m} \bar{z}^{n}-m(z-1)-n(\bar{z}-1)-1 \\
& =\left(\frac{1}{z}\right)^{-m} \bar{z}^{n}+m\left(\frac{1}{z}-1\right)-n(\bar{z}-1)-1-m \frac{(1-z)^{2}}{z},
\end{aligned}
$$

which means that the functions

$$
z \mapsto 1-z^{m} \bar{z}^{n}+m(z-1)+n(\bar{z}-1)
$$

and $z \mapsto 1-\operatorname{Re} z^{m} \bar{z}^{n}$ are $\mu$-integrable.

The other cases are proved in a similar way. It follows that we can choose the function $L: \mathbb{Z}^{2} \times(T \backslash\{1\}) \rightarrow \mathbb{C}$ defined by $L((m, n), z)=(m-n) \operatorname{Im} z$ as a Lévy function for $\mathbb{Z}^{2}$, and that $\mathcal{M}\left(\mathbb{Z}^{2}\right)=\{\mu$ positive Radon measure on $T \backslash\{1\} \mid$ the function $z \mapsto 1-\operatorname{Re} z$ is $\mu$-integrable $\}$.

Now the proposition is a particular case of the theorem given in Section 2. 


\section{REFERENCES}

[1] C. Berg, J. P. R. Christensen and P. Ressel, Harmonic analysis on semigroups, Springer, New York, Heidelberg and Berlin, 1984. MR 86b:43001

[2] W. Bloom and P. Ressel, Positive definite and related functions on hypergroups, Canad. J. Math. 43 (1991), 242-254. MR 92i:43004

[3] H. Buchwalter, Formes quadratiques sur un semi-groupe involutif, Math. Ann. 271 (1985), 619-639. MR 86i:43008

[4] H. Buchwalter, Les foncitons de Lévy existent!, Math. Ann. 274 (1986), 31-34. MR 87e:43007

[5] P. H. Maserick, A Lévy-Khinchin formula for semigroups with involution, Math. Ann. 236 (1978), 209-216. MR 58:12209

Department of Mathematics, Chalmers University of Technology and Göteborg UniVersity, S-412 96 Göteborg, Sweden

E-mail address: dragu@math.chalmers.se 